\title{
Sistema de trazabilidad de la cadena de suministro agroalimentario para cooperativas de frutas y hortalizas basado en la tecnología Blockchain
}

\section{Juan Diego Borrero}

RESUMEN: Blockchain se ha convertido en una tecnología disruptiva que tiene la capacidad de transformar la industria agroalimentaria, puesto que promete resolver muchos problemas relacionados con la falta de confianza en la trazabilidad y el producto que adquieren los consumidores. Sin embargo, las partes implicadas en la cadena de suministro de productos agroalimentarios son numerosas y están físicamente dispersas, lo que dificulta el manejo de datos e información. Como resultado, el proceso de producción no es transparente y la confianza es difícil de construir.

Para ser más exitosas en la economía globalizada actual, las cooperativas deben focalizarse en ofrecer mayor transparencia. Este documento propone un sistema de trazabilidad para una cooperativa agrícola basado en la tecnología blockchain, para resolver la crisis de confianza en la cadena de suministro de los productos agroalimentarios. La aplicación de técnicas de blockchain a la trazabilidad del producto agrario no solo amplía el dominio de la aplicación de la tecnología blockchain, sino que también apoya la generación de confianza entre los diferentes agentes de la cadena. Se exploran las posibles implicaciones del blockchain para los alimentos frescos mediante el desarrollo de una prueba de concepto ( $\mathrm{PoC}$ ) en el ámbito de la trazabilidad agroalimentaria, incorporando, además, la tecnología de los contratos inteligentes. Los hallazgos de la investigación contribuyen a una mejor comprensión de la tecnología blockchain para las diversas partes interesadas en la cadena de alimentos frescos, especialmente para las cooperativas, siendo una oportunidad para la mejora de la reputación y competitividad en una economía altamente globalizada.

PALABRAS CLAVE: Blockchain, Trazabilidad, Cooperativas, Contrato inteligente, Prueba de concepto, Cadena de bloques, Seguridad alimentaria, Cadena de suministro de alimentos frescos.

CLAVES ECONLIT: D82, L29, O33, Q11, Q13, Q16, P13.

Cómo citar este artículo / How to cite this article: BORRERO, J.D. (2019): "Sistema de trazabilidad de la cadena de suministro agroalimentario para cooperativas de frutas y hortalizas basado en la tecnología Blockchain", CIRIECEspaña, Revista de Economía Pública, Social y Cooperativa, 95, 71-94, DOI: 10.7203/CIRIEC-E.95.13123.

Correspondencia: Juan Diego Borrero, Escuela Técnica Superior de Ingeniería, Universidad de Huelva. Investigador Responsable del Grupo de Investigación SEJ-110 ‘Economía Agraria'. Campus ‘El Carmen', Avda. de las Fuerzas Armadas, s/n. 21007, Huelva, Spain. E-mail de contacto: jdiego@uhu.es. 


\section{EXPANDED ABSTRACT}

\section{Agri-food Supply Chain Traceability for Fruit and Vegetable Cooperatives Using Blockchain Technology}

\section{Objectives}

Blockchain is considered today a disruptive technology that has the capacity to transform the agri-food industry, since it promises to solve many problems related to the lack of trust in the product that consumers acquire. However, the parties involved in the agrifood supply chain are numerous and physically dispersed, which makes data and information management difficult. As a result, the production process is not transparent and trust is difficult to build.

In the horticultural sector, technological innovation in traceability is crucial from a economical point of view. An increasing demand in society for greater information about food reflects the need for more transparency. At the same time, more and more fresh products are accompanied by a variety of certification schemes, with an increasing risk of fraud and adulteration. Within this context, cooperatives of fresh fruits and vegetables can gain additional benefits from the implementation of blockchain technology.

This paper seeks to make an initial contribution to the emerging public debate on this issue by providing an overview of blockchain and its application in agriculture, examining implications for food transparency and identifying some potential challenges for agri-food cooperatives.

Based on the above reasons, the main purpose of this article is to conceptualize, design and test a traceability system of the agri-food supply chain based on blockchain technology that helps agri-food cooperatives to improve transparency regarding the origin and the processes incorporated into the product.

\section{Methodology}

A new model that involves blockchain and a smart contract to coordinate the tracking of food in the agriculture supply chain is presented in this paper.

The food supply chain involves many different actors such as farmers, manufacturers, logistics, wholesalers, resellers, retailers and supermarkets. This system is currently inefficient and unreliable. 
In the current situation, much of the data and information on compliance are recorded on paper or stored in a centralized database.

Through the development of a Proof of Concept (PoC) in the field of agri-food supply chain traceability, the possible implications of the blockchain are explored.

The PoC based on a case related to the berries of southern Spain is developed through the construction of a demonstrator that monitors the different nodes involved in the supply chain. The demonstrator is based on a previous analysis of the berries supply chain and the interactions between farmers, the cooperative, its certifiers, logistics providers, retailers and supermarkets, in order to allow a digital representation of a batch of berries to be associated with a unique and immutable digital certificate.

To prove the origin and the traceability of the berries production and to be able to customize the roles of each actor in the supply chain with blockchain, the PoC concept was developed in a Spanish farmers cooperative with a permissioned ledger (hyperledger) and a smart contract.

\section{Results}

At the moment, few would question the relevance of blockchain to agri-food. The main question of adopting blockchain technology in the traceability of goods in the agri-food supply chain, is related to the added value compared to existing ICT (Information and Communication Technologies) solutions. This PoC has shown that it is feasible to incorporate basic information from the field and manufacturing processes, packaging and shipping into a chain of blocks with an authorized general ledger and a smart contract. Compared to traditional situations with centralized databases, the PoC demonstrator shows how blockchain can be used to ensure that different agents share the same level of information about the validity and provenance of inviolable certificates.

This new supply chain architecture via blockchain can provide the relevant data to the participants, it can maintain confidentiality and can spread data effectively among the participants that use the blockchain technology. Therefore, the PoC pilot demonstrated that the blockchain technology could be used successfully in that context.

Furthermore, given the experimentation carried out, it was shown that a medium-sized server (eg VPS in cloud) could function properly as a node for the purposes of this type of use case. There was also no need for special hardware, and the software infrastructure (eg Hyperledger Fabric) is open source and for free.

During the development of the PoC, it was learned that it is important to maintain periodic dialogues between stakeholders in order to achieve a better understanding of the interests of each 
one and to identify common bases for the application of blockchain. The experience in this project shows that the meetings, in which different stakeholders presented and debated different use cases, are a good mechanism for expanding the agri-food blockchain ecosystem.

\section{Conclusions}

The advantage of this model over the centralized models is that all the products are tracked with blockchain and with this traceability it is possible to give confidence to the final consumers about the origin of the products because all the data and transactions carried out in the chain are recorded in the blockchain and managed through a smart contract. The PoC shows that this system is efficient, safe, transparent and avoids intermediaries, which results in lower costs while generating greater confidence in retailers, supermarkets and consumers, which is particularly beneficial for small farmers and cooperatives

The research findings contribute to a better understanding of the blockchain technology for the various stakeholders in the fresh food chain, especially for cooperatives, being an opportunity for the improvement of reputation and competitiveness in a highly globalized economy.

Several limitations were found for the PoC demonstrator. First, the amount of data and information that is shared is very limited. As with most PoC pilots, the project has not yet been able to demonstrate how the system would work when handling a large number of transactions. In addition, our demonstrator focused on a single smart contract. In practice, however, multiple smart contracts would need to be implemented for the different contractual relationships and to keep the transaction data visible only to the relevant subset of participants. Mistakes in the smart contract design have also shown that testing, validation and rigorous semantics are essential to avoiding significant damage to business relationships.

Finally, to take full advantage of the power of blockchain, the adoption of this technology by several cooperatives is recommended. Most cooperatives are too small or have a lack of knowledge to invest in blockchain by themselves.

In summary, blockchain is a promising technology for the competitiveness of cooperatives. The near future will show if blockchain technology will become a reliable and transparent way to provide a differential value for agri-food cooperatives.

KEYWORDS: Blockchain, Food Traceability, Cooperatives, Smart Contract, Smart Agriculture, Trust-building, Agriculture Supply Chain, Proof of Concept. 


\section{Introducción}

Con el desarrollo de la economía mundial, el nivel de vida de las personas ha mejorado. Ello ha provocado un cambio en los hábitos de consumo, prestándose ahora más atención a la seguridad e integridad alimentarias. Sin embargo, en los últimos años, una serie escándalos relacionados con la seguridad alimentaria (Trienekens y Zuurbier, 2008) y la autenticidad de las numerosas certificaciones alimentarias existentes (Boyacia et al., 2014) ponen en peligro la confianza del consumidor en los productos que adquiere (Trienekens et al., 2012).

La agricultura y las cadenas de suministro de alimentos son dos áreas muy interrelacionadas, ya que los productos de la agricultura casi siempre se utilizan como insumos en algunas cadenas de suministro, donde el consumidor suele ser el cliente final.

La cadena de suministro de alimentos involucra a numerosos y diferentes actores, tales como agricultores, procesadores, transportistas, centrales de compra, distribuidores y supermercados. Este sistema es actualmente ineficiente y poco fiable. Por ejemplo, cuando las personas compran productos, no conocen su origen, más allá de la información que aparece en la etiqueta. Se han identificado varias iniciativas, donde la tecnología podría usarse para resolver problemas prácticos de la cadena de suministro agrícola. Estas iniciativas se pueden dividir en dos categorías: integridad alimentaria y apoyo a los pequeños agricultores.

La integridad alimentaria, entendida como la seguridad y autenticidad de los alimentos en la cadena de suministro, tanto en la capa física como en la digital, trata de un intercambio fiable de alimentos a través de la cadena, donde cada actor debe entregar detalles completos sobre el origen de los productos. Es en esta última capa donde se debería proporcionar información fiable sobre el origen y procedencia de los productos alimenticios.

En la situación actual, gran parte de los datos e información sobre el cumplimiento son registrados en papel o almacenados en una base de datos centralizada. Los problemas de estos procesos de información físicos y digitales centralizados son:

- El alto costo y la ineficiencia de los procesos basados en papel.

- El fraude, corrupción y errores en ambos procesos.

- Los problemas debidos a errores humanos y de manipulación de datos (integridad de registros digitales).

- El gasto en certificaciones.

Varios autores han propuesto que el sector de la agricultura pueden ser un área de interés para la aplicación de la tecnología blockchain, puesto que los consumidores están cada vez más preocupa- 
dos por la seguridad y la sostenibilidad de los alimentos y demandan más información sobre la cadena agroalimentaria. Sin embargo, la longitud y la complejidad de las cadenas agroalimentarias modernas han creado una distancia entre consumidores y productores que hace que no sea factible para aquéllos abordar sus preocupaciones y preguntas directamente a los productores. Así, el aumento de la demanda de información sobre alimentos refleja la necesidad de una mayor transparencia. Al mismo tiempo, cada vez más productos alimenticios y bebidas vienen acompañados por una variedad de esquemas de certificación, con un creciente riesgo de fraude (por ejemplo, la venta de productos no calificados con etiquetas o declaraciones de alta calidad) y adulteración.

En este sentido, blockchain, que ofrece transacciones permanentes e inmutables y el acceso a datos distribuidos, tiene el potencial de facilitar el intercambio de datos y reducir las oportunidades de fraude 0 adulteración, aportando mayor transparencia y confianza al consumidor.

Existen numerosos ejemplos de empresas e iniciativas que buscan mejorar la integridad de la cadena de suministro de alimentos a través de la tecnología blockchain como Cargill Inc (Bunge, 2017), Coca-Cola (Chavez-Dreyfus, 2017), Carrefour (Love y Somerville, 2018), Wallmart (Wass, 2017) o la plataforma china Jd.com (Peter, 2017).

Por otro lado, el núcleo de los problemas que plantea la internacionalización para las cooperativas surge de las dificultades y barreras para crecer debido a la pequeña dimensión de las explotaciones que las integran (McMurtry y Reed, 2009). Según la literatura general, la internacionalización ha convertirse en una estrategia indispensable para que las empresas sigan siendo competitivas en los mercados, especialmente en situaciones de crisis económica (Lee y Makhija, 2009). En el caso de las cooperativas, varios autores argumentan que la internacionalización representa una oportunidad para que las cooperativas crezcan y alcancen el tamaño necesario para competir en el contexto globalizado actual, así como para mejorar su eficiencia y posición competitiva en los mercados. Nuestra investigación sugiere que las cooperativas solo pueden ser eficientes y exitosas en la economía globalizada actual si se focalizan de manera consistente en ofrecer transparencia y confianza (Bretos y Marcuello, 2017).

La tecnología blockchain es, probablemente, la más disruptiva desde la llegada de internet y es capaz de transformar las industrias al descentralizar la confianza, generando un intercambio de bienes y servicios sin necesidad de terceros. Esto es posible gracias a que la información que circula a través de los registros contables compartidos está verificada, al igual que quienes se encuentren en los extremos.

La información presente en esta tecnología se introduce mediante un registro inalterable (garantizado mediante criptografía), que se replica en todos los participantes, mediante un protocolo de comunicaciones estándar y que es accesible a todos ellos, eliminando la necesidad de acuses de recibo y conciliaciones. 
La tecnología blockchain puede, por lo tanto, contribuir de manera significativa a conseguir el objetivo de transparencia que lleve a las cooperativas a tener una mejor reputación en los mercados y mejorar su posición competitiva.

En base a los motivos anteriores, el propósito principal de este artículo es conceptualizar, diseñar y probar un sistema de trazabilidad de la cadena de suministro agroalimentaria basado en la tecnología blockchain que ayude a los productores hortofrutícolas asociados en cooperativas a mejorar la transparencia sobre el origen y los procesos incorporados al producto, así como ofrecer, tanto confianza a los compradores frente a los productores de países emergentes de África, América y Asia, como reducir las ventajas comparativas con respecto de los países productores centroeuropeos.

La tecnología blockchain puede proporcionar un medio para asegurar la permanencia de los registros y, potencialmente, facilitar el intercambio de datos entre los diversos actores en la cadena de valor agroalimentaria. Este potencial puede conducir a un cambio de paradigma que facilite la transparencia y la confianza del consumidor en los pequeños productores agrupados en cooperativas.

Hasta donde sabemos, hay pocas publicaciones que apliquen la tecnología blockchain a un sistema de trazabilidad para resolver el problema de la credibilidad de la información en la cadena de suministro agroalimentaria. En este sentido, en este estudio se desarrolla un sistema de trazabilidad de la cadena de suministro agroalimentaria basado en la tecnología blockchain y se exponen los detalles para su implementación. Este sistema puede aumentar la transparencia de la gestión de la cadena de suministro y fortalecer significativamente la confianza en los productos agroalimentarios comercializados.

El resto del documento está organizado de la siguiente manera. Comenzamos con una breve investigación de la literatura existente sobre la tecnología blockchain y su aplicación en la cadena de suministro agroalimentaria. Luego presentamos el marco conceptual del sistema de trazabilidad y describimos la Prueba de Concepto (PoC). Finalmente, discutimos los hallazgos y exponemos las conclusiones y recomendaciones sobre la aplicación de la tecnología blockchain para el sector cooperativo agroalimentario.

\section{Revisión de la literatura}

A nivel mundial, tanto la producción de alimentos como los canales minoristas están cambiando. Existe una creciente dependencia de las cadenas de suministro globales y de los sistemas de distribución a gran escala, como los supermercados. Los sistemas alimentarios son también cada vez más intensivos en capital y están más concentrados e integrados verticalmente. Estas cadenas de sumi- 
nistro aumentan las barreras para que los pequeños productores y procesadores agrícolas participen en los mercados mundiales.

En este contexto, blockchain y los contratos inteligentes constituyen una oportunidad real para una mayor participación en el mercado para los pequeños agricultores y las cooperativas (Tripoli y Schmidhuber, 2018). Ejemplos de aplicaciones se encuentran en las experiencias de AgriLedger, FarmShare, OlivaCoin o Grass Roots Farmers.

Pero las cadenas de suministros agrícolas tienen sustanciales ineficiencias, que afectan a todos los actores, desde productores hasta consumidores. Los retos pasan por mejorar la transpaencia debido a la inconsistencia o no disponibilidad de datos, la alta proporción de trabajo manual y de papel y la falta de interoperabilidad entre los agentes de la cadena.

Para restaurar la confianza del consumidor a raíz de las alertas en seguridad alimentaria, se están tomando muchas medidas en relación a la transparencia en la cadena de suministro agroalimentaria (Akkerman et al., 2010). En este sentido, el concepto de trazabilidad de los alimentos como "la capacidad de rastrear y seguir un alimento en todas las etapas de producción, procesamiento y distribución" (European Commission, 2013) cobra un gran significado.

Varios estudios consideran la utilidad de la adopción de tecnologías avanzadas en la gestión de la trazabilidad agroalimentaria, especialmente con el uso de etiquetas RFID, códigos de barras o QR y su integración con otras tecnologías como WSN (Redes de Sensores Inalámbricos), GPS (Sistema de Posicionamiento Global), GIS (Sistema de Información Geográfica) (Li, Kehoe y Drake, 2006; Li, Lin y Chen, 2010; Sari, 2010; Ustundaga y Tanyasb, 2009; Wang et al., 2010; Yang et al., 2008) o loT (Internet de las Cosas) (Lin et al., 2017a; Zinas et al., 2017).

Actualmente, la tecnología blockchain ha llamado la atención del sector de la Agricultura. Blockchain es una base de datos distribuida formada por bloques unidos en orden cronológico (Morris, 2016; Nakamoto, 2008; Popper, 2016). Son como libros contables (un 'libro mayor') que permiten que múltiples partes accedan y actualicen una única versión mientras mantienen el control compartido. Así, en una red de negocios, como puede ser la de la cadena de alimentos frescos, cualquier grupo de organizaciones, minoristas, productores o consumidores se conecta con el deseo de transferir activos digitales obteniendo, cada miembro, un registro de la transacción y acceso a la última versión del libro mayor. Así, el sistema blockchain establece la confianza en todo el sistema mediante el uso de un libro mayor distribuido que contiene el estado actual de los activos y el historial de todas las transacciones que están protegidas con criptografía para que no se puedan manipular o eliminar.

Al blockchain se le puede incorporar un contrato inteligente (smart contract), que es un programa informático que ejecuta acuerdos establecidos entre dos o más partes, haciendo que ciertas acciones sucedan como resultado de que se cumplan una serie de condiciones específicas (Kosba et al., 2016). Así, en un contrato inteligente se programa las condiciones, se firma por las partes implicadas y se 
'coloca' en un blockchain para que no pueda modificarse. Cuando se da una condición programada, el contrato inteligente ejecuta automáticamente la cláusula correspondiente, pero si una parte no cumple con los términos, el contrato no se ejecuta.

Aunque la tecnología blockchain tiene un potencial considerable para aplicaciones comerciales (Gartner, 2017), no obstante, todavía las innovaciones en arquitecturas blockchain y modelos de negocio asociados al sector agroalimentario no son muy conocidas (Lin et al., 2017b; Tripoli y Schmidhuber, 2018; Yan y Jia, 2017).

En el ámbito de la trazabilidad y blockchain encontramos algunos estudios que procuran resolver los problemas de seguridad alimentaria (Ly y Chen, 2016) y de cumplimiento con los sistemas de APPCC (Tian, 2017), así como aquellos que combinan blockchain con tecnologías RFID (Tian, 2016) o dispositivos loT (Caro et al., 2018, Lin et al., 2018; Shen, Miau y Liu, 2017; Yan y Jia, 2017; Zinas et al., 2017).

Finalmente, en el ámbito de los pequeños productores, la cooperativa The Grass Roots Farmers Cooperative (Grass Roots Farmer's Cooperative, 2017) que vende cestas de carne por suscripción, usa la tecnología blockchain para informar a sus consumidores de una manera fiable sobre las condiciones de cria de sus animales.

\section{Metodología}

Un sistema de trazabilidad agroalimentario pretende registrar toda la información relacionada con la cadena de suministro desde antes del cultivo hasta la distribución de los productos y asociarla a otros datos como pueden ser los ambientales o certificaciones. Como se ha descrito anteriormente, las características de la tecnología blockchain se ajustan perfectamente a las necesidades del sistema de trazabilidad agroalimentario. El objetivo de desarrollar un sistema de trazabilidad agrícola basado en blockchain es aportar una mayor transparencia y generar confianza en los distribuidores, supermercados, consumidores y autoridades para aumentar la competitividad de los pequeños cooperativistas. Esto implica involucrar a diferentes agentes (Ge y Brewster, 2016). Es pertinente traducir la aplicabilidad teórica del blockchain a escenarios reales mediante casos de uso porque todavía siguen existiendo muchos desafíos, algunos técnicos, como la escalabilidad y el rendimiento; y otros no tan técnicos, como los de la legalidad y la gobernanza, que hay que resolver.

El caso de uso en la cadena de suministro de los berries de una cooperativa Huelva (Andalucía, España) involucra problemas de información supuestamente abordados por el blockchain. Desde un 
invernadero de Huelva, los berries recorren un largo camino antes de llegar al consumidor europeo. Hay mucho que los consumidores ( $y$, por lo tanto, también los minoristas) desearían saber sobre los berries. ¿Son alimentos seguros? ¿se producen de manera sostenible? ¿en qué tipo de suelo crecieron las plantas? ¿qué tipo de fertilizante se aplicó? ¿cuáles fueron las condiciones laborales en la explotación? ¿podemos estar seguros de su seguridad y sostenibilidad si llevan un certificado? ¿cómo podemos estar seguros de que el certificado es auténtico?

En relación al blockchain, deben formularse otras preguntas, tales como ¿qué problemas resuelve? ¿qué nuevos problemas crea? ¿quién se beneficia? ¿qué impacto tendrá esta nueva tecnología en los ecosistemas digitales para la transparencia y la confianza en la agroalimentación? ¿este nuevo tipo de confianza y transparencia revolucionará la forma en que se organizan las cadenas agroalimentarias? ¿qué conocimiento y experiencia se necesitan para aprovechar el poder de la cadena de bloques?

Para mejorar la comprensión de la tecnología y sus implicaciones, estas preguntas solo pueden responderse mediante la exploración conjunta de las partes interesadas en un estudio piloto. Gracias a la colaboración con la spin-off Bo True activities, ya se conocían muchos detalles sobre la cadena y la red de suministro de los berries onubenses, por lo que el proyecto podría centrarse en la aplicación de la tecnología blockchain al proceso generado por una cooperativa de pequeños productores de berries de Huelva (España).

La fase preliminar fue identificar las dificultades que debían abordarse antes de explotar comercialmente blockchain, como:

- resolución de los problemas de privacidad y seguridad en las transacciones digitales e intercambio de datos;

- elección entre diferentes diseños e implementaciones blockchain;

- relación e interacción de la base de datos centralizada existente, con la tecnología de red descentralizada;

- decisión sobre la accesibilidad a los datos a falta de un marco regulatorio para el blockchain;

- decisión sobre los problemas relacionados con el rendimiento (velocidad, fiabilidad) y la escalabilidad (capacidad de la cadena de bloques) de la tecnología blockchain;

- decisión sobre los costes derivados de desarrollar y mantener la red de blockchain.

Teniendo en cuenta estas cuestiones, el proyecto aborda los siguientes problemas técnicos para el caso de uso específico:

- ¿Qué problemas relacionados con el intercambio de información, la privacidad y la seguridad existen en el caso de uso?

- ¿Qué datos se deben registrar?

- ¿Qué plataforma de blockchain es la más apropiada para este caso?

- ¿Qué características de la plataforma elegida son más relevantes para el caso de uso?

- ¿Qué partes interesadas se ven afectadas por blockchain? 
- ¿Qué pasos son necesarios para implementar blockchain?

- ¿Qué requisitos específicos en la infraestructura de alojamiento de datos se requieren?

El proyecto adopta un enfoque de múltiples actores con el objeto de obtener una experiencia integral en el desarrollo de aplicaciones blockchain en agroalimentación, así como una perspectiva de experiencia de usuario.

Para decidir la elección de la tecnología blockchain en el proyecto piloto, también se tuvieron en cuenta los siguientes aspectos:

- Factibilidad: determinada por los costes (incluido el hardware mínimo requerido, los costos de licencia, los costos de programación, etc.) y la complejidad de la tecnología.

- Comodidad: determinada por la disponibilidad de software, experiencia y facilidad de implementación.

- Rendimiento: determinado por el número de participantes permitidos, velocidad y tiempo de procesamiento.

Por último, se debía utilizar una ontología y, aunque no conocíamos ninguna relacionada con cadenas de bloques, se escogió la propuesta por Kim, Fox y Gruninger (1995) que ha sido parte clave en el modelado empresarial (Fox y Gruninger, 1998) y que ha despertado el interés de los investigadores en ciencias alimentarias (Dabbene, Ga y Tortia, 2014; Regattieri, Gamberi y Manzini, 2007) para garantizar la seguridad alimentaria a lo largo de la cadena de suministro de alimentos. Las suposiciones clave en el desarrollo de esta ontología para su aplicación a un sistema de bloques son que debe ser posible trazar desde una entidad a otra, y que la unidad de recursos rastreables (también conocida como TRU, una representación de un lote de algo, por ejemplo, de fresas) debe ser rastreable.

Los elementos clave de la tecnología que se manejarán en la PoC son:

- Cadena de bloques: Cada bloque contiene los datos de todas las transacciones dentro de un período de tiempo y puede crear una impresión digital que se puede usar para verificar la validez de la información y conectarse con el siguiente bloque.

- Hashes encadenados: Los bloques están vinculados entre sí en un orden cronológico lineal (como una cadena) con un hash o "resumen" digital del bloque anterior. Esto es clave para la seguridad de blockchain y la garantía de permanencia, ya que cualquier cambio en los datos de un bloque afectaría a todos los demás bloques que siguen.

- Algoritmo de consenso: Para que un nuevo conjunto de transacciones se escriba en un bloque, el bloque debe ser validado por un algoritmo de consenso ${ }^{1}$.

1.- Existen varios algoritmos de este tipo, el más común es la 'prueba de trabajo' (POW), donde un nodo debe resolver un rompecabezas criptográfico que le da derecho a validar el nuevo bloque. Otros algoritmos de consenso incluyen la 'replicación tolerante a fallos bizantina' (Vukolić, 2015) y la 'prueba de participación' (POS y DPOS). 
- Una red de igual a igual: Una red de pares o 'nodos' que, generalmente, proporciona el poder de cómputo para lograr el consenso.

- Proceso de trabajo: Para poder escribir en la 'cadena de bloques', cada nodo necesita completar un complejo procedimiento matemático llamado 'minería'.

- Un archivo distribuido inmediatamente replicado: Cada cadena de bloques se replica en todos los 'nodos' u ordenadores en la red "peer to peer" de esa cadena de bloques. La presencia 0 ausencia de un nodo particular (por ejemplo, estar fuera de línea) no afectará al funcionamiento de la cadena de bloques como un todo.

- Firmas criptográficas: Para saber qué persona (utilizando una identidad expresada como un número) realiza una operación, cada operación se firma usando claves públicas y privadas. Así, todas las transacciones en un blockchain se firman criptográficamente para probar la identidad, la autenticidad y hacer cumplir los derechos de acceso de lectura/escritura.

- Blockchains autorizados o no autorizados: Una cadena de bloques autorizada tiene un conjunto de propietarios que controla los derechos de lectura/escritura/minería.

- Contratos inteligentes: Programas simples que se añaden al blockchain (Buterin et al., 20142016).

La cadena de valor agroalimentaria que probamos en el caso de uso incluye las fases de cultivo, recolección, procesamiento, almacenamiento y distribución, y usa códigos de barras, QR y RFID para la adquisición, procesamiento y control de los datos. Además, se utiliza la tecnología blockchain y el contrato inteligente para garantizar que la información que se comparte y que se publica sea fiable y auténtica.

En el marco de un sistema centralizado de trazabilidad, los miembros de la cadena de suministro confían en un centro de supervisión de la información para transferir y compartir su información. Este sistema de trazabilidad centralizado implementa, efectivamente, el intercambio de información y, en cierta medida, realiza la gestión de la trazabilidad en toda la cadena, pero se trata de un sistema de información monopólico, asimétrico y opaco, que podría generar problemas de confianza, por fraude y falsificación de información. Como se muestra en la Figura 1, el sistema de trazabilidad que incorpora la tecnología blockchain y contratos inteligentes, podría convertirse en una innovación que podría aumentar la transparencia de la cadena de suministro, fortalecer la credibilidad de la información, realizar el seguimiento en tiempo real de los productos agroalimentarios y, en consecuencia, mejorar la seguridad de la cadena de suministro agroalimentaria. 


\section{Figura 1. Esquema de diagrama de flujo y elementos de recogida y almacenamiento de datos en un diseño de Blockchain}

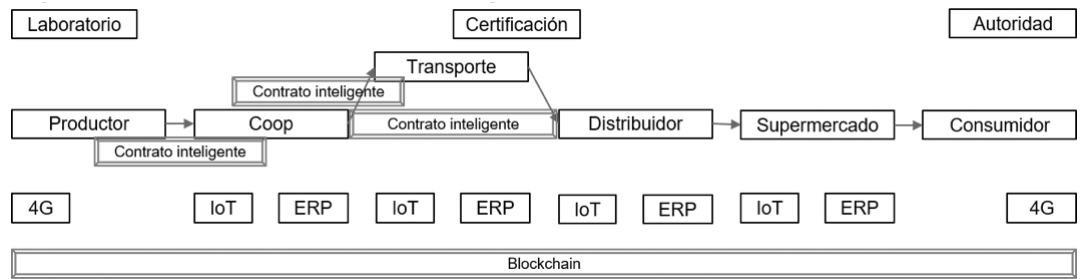

\section{Resultados}

4.1. Agentes y datos que intervienen en el sistema de trazablidad de la cadena de suministro agroalimentaria basada en la tecnología Blockchain

La prueba de concepto basada en un caso de uso relacionado con los berries del sur de España se ha desarrollado mediante la construcción de un demostrador que realiza un seguimiento de los diferentes nodos involucrados en la cadena de suministro de berries frescos. El demostrador se ha basado en un análisis previo de la cadena de suministro de berries frescos y las interacciones entre agricultores, la cooperativa, sus certificadores, proveedores logísticos, distribuidores y supermercados para permitir que una representación digital de un lote de berries se asocie con un certificado digital.

El caso de uso se puede resumir de la siguiente manera: La fruta que se cultiva y recolecta en una finca en Huelva-Andalucía (España), necesita una autoridad de certificación para confirmar que este es el caso y expedir la autorización de firma de un certificado GlobalGap de buenas prácticas agrícolas, lo que permite que la finca certifique los envases individuales de berries que produce y la cooperativa comercializa. Asimismo, el productor y asesor técnico registran, a través de su Smartphone, todas las operaciones realizadas en la finca. Si la finca usa algún tipo de pesticida no autorizado, el laboratorio introduce el dato en la cadena de bloques; si el contador digital registra un consumo de agua, lo inserta en la cadena. Los envases recolectados se identifican utilizando un código de barras, QR o etiqueta RFID (incluyendo información de la finca, variedad, fecha de plantación, condición de fertilización, uso de plaguicidas, etc.) que la cooperativa puede leer y transferir a su ERP. La fruta procesada en la cooperativa se introduce en cámaras a la espera de su expedición y carga. Mientras tanto, 
los sensores monitorizan en tiempo real el estado del producto y del entorno. Toda la información relevante se registra en el sistema blockchain a través de la red 4G para los smartphones, LPWAN para los dispositivos IoT, o LAN para los sensores o datos del ERP.

Así, el ecosistema incluye datos del ERP de la cooperativa como de otros dispositivos loT instalados en el campo que miden datos ambientales, de suelo y planta, etiquetas y códigos 2D y sensores en la central de manipulación que monitorean el estado del producto que pasa por sus diferentes fases de manipulación, conservación y expedición. Toda esta información es verificada por el sistema blockchain sin ninguna intervención humana y se abre a todos los miembros autorizados. Así, todas las partes involucradas en esta cadena pueden verificar la validez del certificado emitido al consultar el blockchain: El retail puede consultar los datos en el portal blockchain y asegurarse que el contrato inteligente se ha ejecutado, y los consumidores pueden usar su Smartphone para acceder a los datos guardados en el blockchain y obtener toda la información de los productos agroalimentarios y su trazabilidad (productor, finca, fecha de recolección, cooperativa, información del procesado, fecha de expedición, tratamientos realizados...) simplemente escaneando el código 2D.

Además, toda la información a lo largo de la cadena de suministro agroalimentaria es totalmente auditable, lo que significa que los distribuidores también pueden obtener información detallada sobre los productos finales en tiempo real inspeccionando el sistema blockchain.

\subsection{Alternativas de diseño e implementación para Blockchain}

Actualmente existe una gran cantidad de tecnologías de software blockchain que reflejan decisiones de diseño diferentes en términos de arquitectura técnica. Además de Bitcoin, las tecnologías blockchain disponibles más populares en este momento son Ethereum (Gerring, 2016), Hyperledger, que ha recibido un amplio apoyo y se está utilizando en muchos proyectos diferentes, incluido su uso por parte de Walmart en la cadena de suministro del cerdo (del Castillo, 2016; Higgins, 2017) y BigChainDB (McConaghy, 2017).

Las elecciones técnicas clave incluyen lo siguiente:

1. Diseño del permiso: Bitcoin y Ethereum asumen libros abiertos, sin permisos, mientras que Hyperledger propone múltiples blockchains, cada uno para un sector diferente.

2. Algoritmo de consenso: Bitcoin y Ethereum usan un algoritmo de consenso de "prueba de trabajo", mientras que Hyperledger y BigChainDB usan métodos de votación más simples.

3. Contratos inteligentes: Tanto Ethereum como Hyperledger han enfatizado la capacidad de ejecutar contratos inteligentes en su stack de tecnología?

2.- Los contratos inteligentes se pueden escribir en diferentes lenguajes de computación. Ethereum proporciona 'Solid' como lenguaje de contrato, e Hyperledger usa 'Go'. 
Debido a que Hyperledger permite configuraciones blockchain para sectores comerciales específicos, como el agroalimentario, así como por la capacidad de programar chaincodes para permitir el acceso a ciertos tipos de datos al contrato inteligente por diferentes participantes, decidimos utilizar esta tecnología para la Prueba de Concepto.

\subsection{Escalabilidad y privacidad de las aplicaciones Blockchain}

La Prueba de Concepto se sometió a pruebas de escalabilidad para determinar, en la práctica, el rendimiento real, no siendo éste un aspecto crítico si se dimensionan bien los puntos de control de trazabilidad.

Con Hyperledger los datos generados por la Prueba de Concepto se incluyeron dentro de un contrato inteligente y estaban disponibles o 'visibles' solo para los participantes en el contrato inteligente. Esto asegura que se mantenga la confidencialidad. Para ello, el acceso a los datos debía estar escrito en el contrato inteligente desde el principio ${ }^{3}$.

\subsection{Grado de aceptación de las partes interesadas}

La naturaleza distribuida de blockchain significa que su implementación inevitablemente involucra a múltiples partes. Las partes interesadas clave en el caso de uso fueron los productores socios, la cooperativa de manipulación, sus empresas logísticas, sus plataformas de compra, sus retailers, la organización de certificación GlobalGap, sus proveedores de servicios, como los laboratorios y las compañías de seguros, y la autoridad de seguridad alimentaria.

Al implementar una solución blockchain para estos interesados, se evaluaron los costos y beneficios de blockchain. Sin embargo, la adopción del blockchain puede cambiar el ecosistema al introducir nuevos jugadores y cambiar la posición de los mismos dentro del ecosistema.

Se detectó un considerable nivel de escepticismo entre las partes interesadas, debido a la forma en que Blockchain se presenta como una panacea para todos los problemas de información, los problemas técnicos que aún quedan por resolver (lansiti y Lakhani, 2017) y, porque no conocen casos de éxito próximos a su realidad.

3.- Otro problema importante con respecto a la privacidad es el Reglamento general de protección de datos de la UE (GDPR). En primer lugar, las cadenas de bloques abiertas sin permiso están completamente descentralizadas y distribuidas, lo que significa que no existe una sola entidad con responsabilidad legal para el procesamiento de datos en la cadena de bloques. En una cadena de bloques autorizada como Hyperledger, en teoría, al menos, hay una entidad responsable de la participación en el Blockchain. En segundo lugar, las cadenas de bloques están diseñadas para ser inmutables, pero de acuerdo con los datos de GDPR, deben eliminarse por completo si la persona relevante lo solicita. Además, las partes específicas del GDPR dependen de la ubicación donde se procesan los datos y, obviamente, los datos de la cadena de bloques se están procesando en todas partes y en ninguna parte. Estos problemas deberán abordarse mediante una combinación de cambios en la regulación y los avances en la tecnología. 
Las principales preocupaciones de nuestros grupos de interés fueron las siguientes:

- La fiabilidad de los datos que se colocan en la cadena de bloques;

- Cómo lidiar con las diferentes tecnologías blockchains;

- La validez y consistencia de los contratos inteligentes;

- La eficacia de blockchain en la prevención real del fraude en la cadena alimentaria.

La mayoría de estas preocupaciones pueden resolverse 0 aliviarse mediante avances tecnológicos (por ejemplo, el uso de datos generados por sensores y máquinas) o institucionales (por ejemplo, el desarrollo de estándares para contratos inteligentes).

Si bien se espera que el uso de blockchain desaliente la manipulación de datos, no podrá evitar que las personas ingresen información falsa o errónea u oculten información en la cadena de bloques, aunque el uso de sensores y dispositivos loT minimizará estos problemas.

Blockchain tampoco puede, por sí solo, eliminar el fraude en la cadena alimentaria, sin embargo, cuando más datos disponibles estén vinculados a blockchain, será más fácil detectarlo, debido a la posibilidad de utilizar controles cruzados y a la inmutabilidad de los registros.

\subsection{Arquitectura}

El prototipo ha sido construido utilizando Hyperledger Fabric (v0.6) que permite a las partes implicadas actualizar y consultar los datos en el blockchain en vista de su rol y derechos de acceso.

En Hyperledger Fabric, un miembro puede ser de diferentes tipos: usuarios, pares y validadores. Los usuarios se pueden dividir en aquellos que necesitan enviar datos a la plataforma, como productores, cooperativa y transportistas, los cuáles deben registrarse; y los usuarios finales, como el supermercado o el consumidor, que pueden consultar y obtener los resultados. Este tipo de usuario normalmente no necesita registrarse. Los pares son nodos que guardan una copia del libro mayor. Los validadores también conservan una copia, pero validan las transacciones entrantes y pueden participar en el algoritmo de consenso de blockchain.

Para evitar que todos los nodos tengan acceso idéntico a todos los datos se diseñó un contrato inteligente que proporcionaba a los participantes del contrato acceso a diferentes datos dentro de esa base de datos. Todas las consultas a los datos estuvieron predefinidas en las funciones escritas en el código del contrato inteligente.

La arquitectura básica del sistema Blockain, comparada con el sistema centralizado, se diseñó como se muestra en la Figura 2. 


\section{Figura 2. Arquitectura del demostrador (arquitectura centralizada vs Blockchain)}
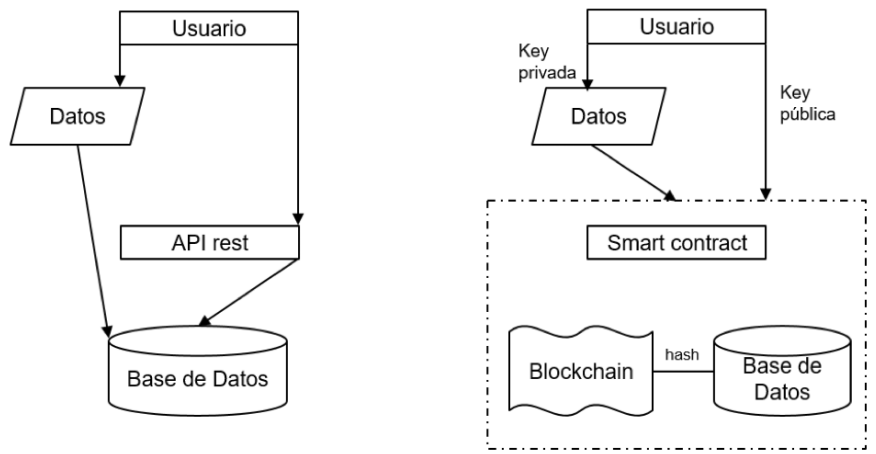

Una ventaja importante de esta arquitectura es que permite un fácil lanzamiento del demostrador y, por lo tanto, puede desplegarse y ampliarse sin demasiado esfuerzo.

Finalmente, para una fácil interacción de los usuarios finales con la cadena de bloques se puede desarrollar una API y una aplicación de front-end accesible desde un PC o dispositivo móvil como la que se presenta en la Figura 3.

\section{Figura 3. Ejemplo de un Front-end para verificar certificados en Blockchain}

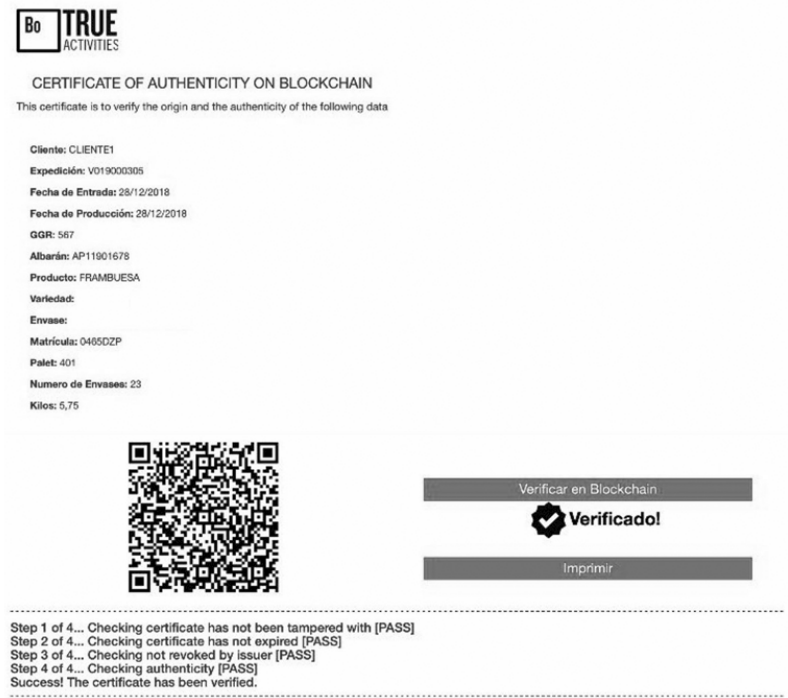




\section{Conclusiones, limitaciones, ventajas, obstáculos y recomendaciones}

La tecnología blockchain ofrece muchos beneficios, ya que puede proporcionar una forma segura y distribuida para realizar transacciones entre diferentes partes. Este es un elemento clave en la agricultura y en la cadena de suministro alimentaria, donde participan numerosos actores (Lin et al., 2017b).

La novedad de este artículo reside en que todos los datos y transacciones efectuadas en la cadena, se registran en la cadena de bloques, gestionada a través de contratos inteligentes. La prueba de concepto demuestra que este sistema es más eficiente, más seguro, más transparente y evita intermediarios, lo que redunda en menores costes para los cooperativistas, a la vez que genera mayor confianza en los distribuidores, supermercados y consumidores, lo que es particularmente beneficioso para los pequeños agricultores y cooperativas.

Para acreditar el origen y trazabilidad de la producción de berries con blockchain se desarrolló una prueba de concepto en una cooperativa onubense con una capa de Hyperledger para hacer una blockchain permisionada y poder customizar los roles de cada actor en la red. La experiencia en consultoría con berries permitió programar adecuadamente los contratos inteligentes de blockchain.

Por el momento, pocos cuestionan la relevancia de blockchain para la agroalimentación. La pregunta principal es más bien sobre el valor agregado de blockchain en comparación con las soluciones TIC (Tecnologías de la Información y Comunicación) existentes. Esta prueba de concepto (PoC) ha demostrado que es factible poner información básica de cuadernos de explotación y datos de manipulación, envasado y expedición en una cadena de bloques con un libro mayor autorizado y un contrato inteligente. En comparación con las situaciones tradicionales con bases de datos centralizadas, el demostrador PoC muestra cómo se puede utilizar blockchain para garantizar que las diferentes partes compartan la misma capa de información sobre la validez y procedencia de los certificados que son inviolables.

Mostramos, por el diseño de la arquitectura, que dicho sistema puede proporcionar los datos relevantes a los participantes, puede mantener la confidencialidad y puede propagar datos de manera efectiva entre los participantes que utilizan la tecnología blockchain. Por lo tanto, la prueba de concepto demostró que la tecnología blockchain podría usarse con éxito en dicho contexto.

Además, dada la experimentación realizada, se demostró que un servidor de tamaño medio (por ejemplo, en la nube) podría funcionar adecuadamente como un nodo para los fines de este tipo de caso de uso. Tampoco hubo necesidad de hardware especial y toda la infraestructura de software (en este caso Hyperledger Fabric) es de código abierto y gratuita. 
Durante el desarrollo de la PoC, se aprendió que es importante mantener diálogos periódicos entre las partes interesadas para lograr una mejor comprensión de los intereses de cada una e identificar bases comunes para la aplicación de blockchain. La experiencia en este proyecto muestra que las reuniones, en las que se debatían diferentes casos de uso, son un buen mecanismo para hacer crecer el ecosistema de blockchain en la agroalimentación. Como las discusiones y consultas con las partes interesadas han dejado claro, blockchain creará diferentes oportunidades y desafíos para diferentes partes interesadas $u$ organizaciones, dependiendo de su posición actual en el mercado y en la cadena de valor de la información alimentaria.

Por el momento, pocas aplicaciones de blockchain relacionadas con casos reales han ido más allá de la fase de 'Prueba de Concepto' o pilotos a pequeña escala (Ge et al., 2017).

Se encontraron varias limitaciones para el demostrador de PoC. En primer lugar, la cantidad de datos e información que se comparte es muy limitada. Al igual que con la mayoría de los pilotos PoC, el proyecto aún no ha podido demostrar cómo funcionaría cuando se maneje una gran cantidad de transacciones. Dado el alcance limitado del proyecto, queda por determinarse cuáles serían las consecuencias de los recursos y la capacidad de producción de nuestro sistema. Además, nuestro demostrador se centró en un único contrato inteligente. En la práctica, sin embargo, se necesitarían implementar múltiples contratos inteligentes para las diferentes relaciones contractuales y para mantener los datos de transacción visibles solo para el subconjunto relevante de participantes. Un aspecto que también debería abordarse es la interacción entre los contratos inteligentes y la viabilidad de los flujos de datos entre los contratos inteligentes. Finalmente, los errores repetidos en el diseño del contrato inteligente han demostrado que las pruebas, la validación y la semántica rigurosa son esenciales para evitar daños significativos en las relaciones comerciales.

Dado el nivel cada vez mayor de digitalización y demanda de información e integridad del producto, el sector agroalimentario se encuentra en una posición única para explorar el potencial de Blockchain. Si bien la aplicación de blockchain en la industria agroalimentaria aún se encuentra en su etapa inicial, se puede esperar que cada vez más organizaciones tomen iniciativas.

Como ventajas de un sistema de trazabilidad de la cadena de suministro agroalimentaria basado en la tecnología blockchain, se tienen:

Beneficio de la gestión de la trazabilidad. El sistema de trazabilidad de la cadena de suministro agroalimentaria crea un tipo de cadena de información agroalimentaria que abarca el regulador de la seguridad alimentaria, el vivero, la finca, la central de manipulación y envasado, la empresa logística, el retail y el consumidor, así como otros dispositivos loT. Confiando en el sistema blockchain, toda la información agroalimentaria en la cadena de suministro es transparente y abierta, por lo que la empresa logística podría implementar un seguimiento en tiempo real para los productos agroalimentarios, el regulador podría ejecutar la gestión de trazabilidad y el consumidor podría obtener la información completa de los productos en toda la cadena de suministro agroalimentaria. 
Beneficio para mejorar la credibilidad de la información sobre seguridad agroalimentaria. Actualmente, la mayoría de los sistemas de trazabilidad de la cadena de suministro se basan en la idea de utilizar un sistema centralizado. Sin embargo, este tipo de organizaciones centralizadas son completamente opacas y el retail o consumidor nunca podrá conocer los detalles internos de las transacciones. Al utilizar blockchain, este sistema de trazabilidad elimina la necesidad de una organización centralizada de confianza y proporciona una plataforma de información para todos los miembros con apertura, transparencia, neutralidad, fiabilidad y seguridad.

Beneficio para luchar contra productos falsos. Los datos de información del producto se podrían encriptar vinculando el producto con un identificador, lo que podría proteger al producto de la falsificación. Dado que este proceso no necesita de ninguna operación manual, disminuye en gran medida los errores causados por factores humanos. Además, al utilizar la tecnología blockchain, los miembros de este sistema no pueden manipular la información agroalimentaria, lo que aumenta aún más la seguridad del producto.

Sin embargo, como tecnología emergente, existen algunos obstáculos para su aplicación como son el alto costo y la inmadurez de la tecnología. Otros problemas están relacionados con el tamaño cada vez mayor de la cadena agroalimentaria, en relación al almacenamiento y sincronización de datos.

Finalmente, para aprovechar al máximo el poder de blockchain, se recomienda la adopción de blockchain por parte de varias cooperativas. La mayoría de las cooperativas son demasiado pequeñas o carecen de la experiencia para invertir en blockchain por sí mismas. Además, dado el desarroIlo actual de blockchain, las incertidumbres son demasiado altas para desarrollar un piloto convincente para las partes individuales. Por lo tanto, es importante abordar los problemas de aplicación en las agendas de investigación públicas. Así, el foco principal de la investigación no está en la tecnología blockchain en sí misma, sino en su aplicación a casos de uso adecuados.

En resumen, blockchain es una tecnología prometedora para la competitividad de las cooperativas. El futuro próximo mostrará si la tecnología blockchain se convertirá en una forma segura, fiable $y$ transparente que aporte un valor diferencial a las cooperativas agroalimentarias. 


\section{Referencias}

AKKERMAN, R., FARAHANI, P. \& GRUNOW, M. (2010): "Safety and sustainability in food distribution: a review of quantitative operations management approaches and challenges", OR Spectrum, 32, 863-904, DOI: 10.1007/s00291-010-0223-2.

BOYACIA, L.H., TEMIZA, H.T., UYSALA, R.S., VELIOGLUC, H.M., YADEGARIA, R.I. \& RISHKANA, M.M. (2014): "A novel method for discrimination of beef and horsemeat using Raman spectroscopy. Food Chern", 148, 37-41, DOI: 10.1016/j.foodchem.2013.10.006.

BRETOS, I. \& MARCUELLO, C. (2017): "Revisiting globalization challenges and opportunities in the development of cooperatives", Annals of Public and Cooperative Economics, 88(1), 47-73, DOI: 10.1111/apce.12145.

BUNGE, J. (2017): "Latest Use for a Bitcoin Technology: Tracing Turkeys From Farm to Table", The Wall Street Journal. Recuperado de: https://www.wsj.com/articles/latest-use-for-a-bitcoin- technology-tracing-turkeysfrom-farm-to-table-1508923801.

BUTERIN, V. et al. (2014-2016): "Ethereum White Paper - A Next-Generation Smart Contract and Decentralized Application Platform". Recuperado de: https://github.com/ethereum/wiki/wiki/WhitePaper.

CARO, M.P., ALI, M.S., VECCHIO, M. \& GIAFFREDA, R. (2018): "Blockchain-based traceability in AgriFood supply chain management: A practical implementation". Presentado en: 2018 IoT Vertical and Topical Summit on Agriculture, Tuscany, Italy, DOI: 10.1109/IOT-TUSCANY.2018.8373021.

CHAVEZ-DREYFUSS, G. (2018): “Coca-Cola, U.S. State Dept to use blockchain to combat forced labor", Reuters. Recuperado de: https://www.reuters.com/article/usblockchain-coca-cola-labor/cocacola-u - s - s tate-dept - to - u se - blockchain - to - combatforced-IaboridUSKCN1GS2PY?mc_cid=d7c996d219\&mc_eid=4c123074ea.

DABBENE, F., GAY, P. \& TORTIA, C. (2014): "Traceability issues in food supply chain management: A review", Biosystems Engineering, 120, 65-80, DOI: 10.1016/j.biosystemseng.2013.09.006.

DEL CASTILLO, M. (2016): "Walmart Blockchain Pilot Aims to Make China's Pork Market Safer CoinDesk", CoinDesk. Recuperado de: http://www.coindesk.com/walmart-blockchain-pilot-chinapork-market/.

EUROPEAN COMMISSION (2013): "EU food market overview", Enterprise and Industry. Recuperado de: http://ec.europa.eu/enterprise/sectors/food/eumarket/index_en.

FOX, M.S. \& GRUNINGER, M. (1998): "Enterprise Modelling", Al Magazine, 19(3), 109-121, DOI: https://doi.org/10.18417/emisa.9.1.2. 
GARTNER (2017): "Top Trends in the Gartner Hype Cycle for Emerging Technologies". Recuperado de https://www.gartner.com/smarterwithgartner/top-trends-in-the-gartner-hype-cycle-for-emergingtechnologies-2017/.

GE, L. \& BREWSTER, C.A. (2016): "Informational institutions in the agri-food sector: meta-information and meta-governance of environmental sustainability", Current Opinion in Environmental Sustainability, 18, 73-81, DOl: https://doi.org/10.1016/j.cosust.2015.10.002.

GE, L., BREWSTER, C., SPEK, J., SMEENK, A. \& TOP, J. (2017): Blockchain for Agriculture and Food; Findings from the pilot study, Wageningen: Wageningen Economic Research, DOI: https://doi.org/10.18174/426747.

GERRING, T. (2016): "Cut and try: building a dream - Ethereum Blog", Ethereum Blog. Recuperado de https://blog.ethereum.org/2016/02/09/cut-and-try-building-a-dream/.

GRASS ROOTS FARMERS' COOPERATIVE (2017): "How we 're using blockchain tech for total transparency". Recuperado de https://grassrootscoop.com/blockchain-tech-for-totaltransparency/.

HIGGINS, S. (2017): "Walmart: Blockchain Food Tracking Test Results Are 'Very Encouraging' CoinDesk", CoinDesk. Recuperado de http://www.coindesk.com/walmart-blockchain-foodtrackingtest-results-encouraging/.

IANSITI, M. \& LAKHANI, K.R. (2017): "The Truth About Blockchain", Harvard Business Review, January-February, 118-127.

KIM, H.M., FOX, M.S. \& GRUNINGER, M. (1995): "An ontology of quality for enterprise modelling". Presentado en: Proceedings 4th IEEE Workshop on Enabling Technologies: Infrastructure for Collaborative Enterprises (WET ICE '95), IEEE Comput. Soc. Press, 105-116.

KOSBA, A. MILLER, E., SHI, Z. \& PAPAMANTHOU, C. (2016): "Hawk: The Blockchain Model of Cryptography and Privacy-Preserving Smart Contracts". Presentado en: IEEE Symposium on Security and Privacy (SP), San Jose, CA, 839-858, DOI: 10.1109/SP.2016.55.

LEE, S. \& MAKHIJA, M. (2009): "Flexibility in internationalization: Is it valuable during an economic crisis?", Strategic Management Journal, 30(5), 537-555, DOI: https://doi.org/10.1002/smj.742.

LI, D., KEHOE, D. \& DRAKE, P. (2006): "Dynamic planning with a wireless product identification technology in food supply chains", International Journal of Advanced Manufacturing Technology, 30, 938-944, DOI: 10.1007/s00170-005-0066-1.

LI, M.B., LIN, Z.X. \& CHEN, C. (2010): "Application of RFID on products tracking and tracing system", Computer Integrated Manufacturing Systems, 16(1), 202-208.

LIN, J., SHEN, Z., MIAO, C. \& LIU, S. (2017a): "Using blockchain to build trusted lorawan sharing server", International Journal of Crowd Science, 1(3), 270-280, DOI: https://doi.org/10.1108/JJCS-082017-0010. 
LIN, J., SHEN, Z., MIAO, C., ZHANG, A. \& CHAI, Y. (2018): "Blockchain and IOT based Food Traceability for Smart Agriculture". Presentado en: Proceedings of 3rdlnternational Conference on Crowd Science and Engineering, Singapur, Julio, DOI: https://doi.org/10.1145/3126973.3126980.

LIN, Y.P., PETWAY, J.R., ANTHONY, J., MUKHTAR, H., LIAO, S.W., CHOU, C.F. \& HO, Y.F. (2017b): "Blockchain: The Evolutionary Next Step for ICT E-Agriculture", Environments, 4(3), 50, DOI: 10.3390/environments4030050.

LOVE, J. \& SOMERVILLE, H. (2018): "Retailer Carrefour using blockchain to improve checks on food products", Reuters. Recuperado de: https://www.reuters.com/article/us-didi-mexico/chineseuberrival-didi-launches-in-mexico-recruits-drivers-idUSKCN1HDOPH.

LV, F. \& CHEN, S. (2016): "Research on Establishing a Traceability System of Quality and Safety of Agricultural Products Based on Blockchain Technology", Rural Finance Research, 12, 22-26, DOI: 10.1109/IVS.2018.8500647.

McCONAGHY, T. (2017): "BigchainDB 2017 Roadmap", The BigchainDB Blog. Recuperado de: https://blog.bigchaindb.com/bigchaindb-2017-roadmap-d2e7123f9874

McMURTRY J. \& REED D. (2009): Co-operatives in a Global Economy: The Challenges of Co-operation across Borders, Newcastle: Cambridge Scholars Press.

MORRIS, D.Z. (2016): "Leaderless, Blockchain-Based Venture Capital Fund Raises \$100 Million, And Counting", Fortune. Recuperado de: http://fortune.com/2016/05/15/leaderlessblockchain-vc-fund/.

NAKAMOTO, S. (2008): "Bitcoin: A Peer-to-Peer Electronic Cash System". Recuperado de: https://bitcoin.org/bitcoin.pdf.

PETER, A. (2017): "In China, You Can Track Your Chicken On-You Guessed It- The Blockchain", Fast Company. Recuperado de: https://www.fastcompany.com/40515999/inchina-you-can-track-yourchicken-on-you-guessed-it-the-blockchain

POPPER, N. (22 mayo 2016): "A Venture Fund With Plenty of Virtual Capital, but No Capitalist", New York Times. Recuperado de: http://www.nytimes.com/2016/05/22/business/dealbook/cryptoetherbitcoin-currency.html?_r=1

REGATTIERI, A., GAMBERI, M. \& MANZINI, R. (2007): "Traceability of food products: General framework and experimental evidence", Journal of Food Engineering, 81(2), 347-356, DOI: 10.1016/j.jfoodeng.2006.10.032.

SARI, K. (2010): "Exploring the impacts of radio frequency identification (RFID) technology on supply chain performance", European Journal of Operational Research, 207, 174-183, DOI: 10.1016/j.ejor.2010.04.003.

TIAN, F. (2016): "An agri-food supply chain traceability system for China based on RFID \& blockchain technology". Presentado en: Proceeding ofthe 13th International Conference on Service Systems and Service Management (ICSSSM'16), 1-6, DOI: https://doi.org/10.1109/ICSSSM.2016.7538424. 
TIAN, F. (2017): "A supply chain traceability system for food safety based on HACCP, blockchain \& Internet of things". Presentado en: International Conference on Service Systems and Service Management (ICSSSM), DOI: 10.1109/ICSSSM.2017.7996119.

TRIENEKENS, J.H. \& ZUURBIER, J. (2008): "Quality and safety standards in the food industry, developments and challengers", International Journal of Prod. Econ, 113, 107-122, DOI: https://doi.org/10.1016/j.ijpe.2007.02.050.

TRIENEKENS, J.H., WOGNUM, P.M., BEULENS, A.J.M. \& VAN DER VORST, J.G.A.J (2012): "Transparency in complex dynamic food supply chains", Advanced Engineering Informatics, 26(1), 55-65, DOI: 10.1016/j.aei.2011.07.007.

TRIPOLI, M. \& SCHMIDHUBER, J. (2018): Emerging Opportunities for the Application of Blockchain in the Agri-food Industry, Roma: FAO y ICTSD.

USTUNDAGA, A. \& TANYASB, M. (2009): "The impacts of Radio Frequency Identification (RFID) technology on supply chain costs", Transportation Research Part E: Logistics and Transportation Review, 45(1), 29-38, DOI: 10.1016/j.tre.2008.09.001

VUKOLIC, M. (2015): "The quest for scalable blockchain fabric: Proof-of-work vs. BFT replication". Presentado en: International Workshop on Open Problems in Network Security, Springer.

WANG, L., KWOK, S.K. \& IP, W.H. (2010): "A radio frequency identification and sensor-based system for the transportantion of food", Journal of Food Engineering, 101, 120-129, DOI: 10.1016/j.jfoodeng.2010.06.020.

WASS, S. (22 Agosto 2017): "Food Companies Unite to Advance Blockchain for Supply Chain Traceability", Global Trade Review. Recuperado de: www.gtreview.com/news/fintech/food-companies-unite-to-advance-blockchain-for-supply-chain-traceability/.

YANG, Y. \& JIA, Z. (2017): "Application and Challenge of Blockchain Technology in the Field of Agricultural Internet of Things", Information Technology, 258, 24-26.

YANG, X.T. \& QIAN, J.P., SUN, C.H. (2008): "Design and application of safe production and quality traceability system for vegetable", Transactions of the Chinese Society of Agricultural Engineering, 24(3), 162-166, DOI: https://doi.org/10.1007/978-3-642-27281-3_43.

ZINAS, N., KONTOGIANNIS, S., KOKKONIS, G., VALSAMIDIS, S. \& KAZANIDIS, I. (2017): "Proposed open source architecture for Long Range monitoring. The case study of cattle tracking at Pogoniani". Presentado en: Proceedings of the 21st Pan-Hellenic Conference on Informatics (PCI 2017), ACM, New York, NY, USA, 57(6), DOI: https://doi.org/10.1145/3139367.3139437. 